\title{
EQUALITY OF GRAPHOIDAL AND ACYCLIC GRAPHOIDAL COVERING NUMBER OF A GRAPH
}

\author{
INDRA RAJASINGH AND P. ROUSHINI LEELY PUSHPAM
}

\begin{abstract}
A graphoidal cover of a graph $G$ is a collection $\psi$ of (not necessarily open) paths in $G$ such that every vertex of $G$ is an internal vertex of at most one path in $\psi$ ad every edge of $G$ is in exactly one path in $\psi$. If no member of $\psi$ is a cycle, then $\psi$ is called an acyclic graphoidal cover of $G$. The minimum cardinality of a graphoidal cover is called the graphoidal covering number of $G$ and is denoted by $\eta$ and the minimum cardinality of an acyclic graphoidal cover is called an acyclic graphoidal covering number of $G$ and is denoted by $\eta_{a}$. In this paper we characterize the class of graphs for which $\eta=\eta_{a}$.
\end{abstract}

\section{Introduction}

The concept of graphoidal cover was introduced by B.D. Acharya and E. Sampathkumar in 1987 and a study of the graphoidal covering number was initiated by them [1]. Since then this area of research has been explored by several authors $[2,3,10,11,12,13$, 19]. The concept of an acyclic graphoidal cover was introduced by Suresh Suseela and pursued by Arumugam et al., [14, 15].

By a graph $G=(V, E)$ we mean a finite, undirected, connected graph without loops or nultiple edges. The order and size of $G$ are denoted by $p$ and $q$ respectively. For graph theroetic terminology we refer to Harary [16].

If $P=\left(v_{0}, v_{1}, \ldots, v_{n}\right)$ is a path or a cycle in $G, v_{1}, \ldots, v_{n-1}$ are called internal vertices of $P$. If $P=\left(v_{0}, v_{1}, \ldots, v_{n}\right)$ and $Q=\left(v_{n}=w_{0}, w_{1}, \ldots, w_{m}\right)$ are two paths in $G$ then the walk obtained by concatenating $P$ and $Q$ at $v_{n}$ is denoted by $P o Q$.

Definition 1.1. A graphoidal cover of a graph $G$ is a set $\psi$ of (not necessarily open) paths in $G$ satisfying the following conditions.

(i) Every path in $\psi$ has at least two vertices.

(ii) Every vertex of $G$ is an internal vertex of at most one path in $\psi$.

(iii) Every edge of $G$ is in exactly one path in $\psi$.

$\psi$ is called an acyclic graphoidal cover of $G$ if no member of $\psi$ is a cycle in $G$. The minimum cardinality of (an acyclic) a graphoidal cover of $G$ is called the (acyclic) graphoidal covering number of $G$ and is denoted by $\left(\eta_{a}\right) \eta$.

Received February 13, 2003; revised May 24, 2004.

2000 Mathematics Subject Classification. 05C.

Key words and phrases. Graphoidal covering number, acyclic graphoidal covering number. 
Definition 1.2. Let $\psi$ be a collection of internally disjoint paths in $G$. A vertex of $G$ is said to be an interior vertex of $\psi$ if it is an internal vertex of some path in $\psi$. Any vertex which is not an interior vertex of $\psi$ is said to be an exterior vertex of $\psi$.

Theorem 1.3.([18]) For any graphoidal cover $\psi$ of $G$, let $t_{\psi}$ denote the number of exterior vertices of $\psi$. Let $t=\min t_{\psi}$, where the minimum is taken over all graphoidal covers of $G$. Then $\eta=q-p+t$ where $p$ and $q$ denote respectively the order and size of $G$.

Corollary 1.4.([18]) For any graph $G, \eta \geq q-p$. Moreover the following are equivalent.

(i) $\eta=q-p$.

(ii) There exists a graphoidal cover without exterior vertices.

(iii) There exists a set of internally disjoint and edge disjoint paths without exterior vertices.

Theorem 1.5.([17]) For any graph $G$ with $\delta \geq 3, \eta=q-p$

Remark 1.6. Results analogous to Theorem 1.3, Corollary 1.4 and Theorem 1.5 are also true for an acyclic graphoidal covering number of a graph.

Theorem 1.7.([15]) For any graph with $\delta \geq 3, \eta=\eta_{a}$.

For graphs with $\delta \leq 2$ it is not necessary that $\eta=\eta_{a}=q-p$. Hence the problem of characterizing graphs with $\delta \leq 2$ satisfying $\eta=\eta_{a}$ is challenging. In this paper we solve this problem. For our further discussion we confine ourselves to graphs with $\delta \leq 2$. We need the following definition and theorems.

Definition 1.8.([7]) Let $\mathcal{G}(f)$ denote the collection of all blocks whose edge set can be decomposed into a cycle $C$ and a collection $\wp$ of internally disjoint paths such that each path $P$ in $\wp$ has $f \in V(C)$ as its origin and $|V(P) \cap V(C)| \leq 2$ (The collection $\wp$ may be empty in which case the corresponding member of $\mathcal{G}(f)$ is a cycle). We observe that if $G \in \mathcal{G}(f)$ and $G$ is not a cycle, then $\operatorname{deg} f=|\wp|+2=\Delta$ and there is at most one vertex $v \neq f$ with $\operatorname{deg} v=\Delta$.

Let $\mathcal{F}$ and $\mathcal{F}_{a}$ denote respectively the class of all graphs $G$ with $\eta=q-p$ and $\eta_{a}=q-p$.

Theorem 1.9.([4]) Let $G$ be a 2-connected graph with $p \geq 3$. Then $G \notin \mathcal{F}$ if and only if $G$ is a cycle or a cycle with exactly one chord or a theta graph.

Theorem 1.10.([4]) Let $G$ be a 2-edge connected graph with $\delta=2$. Then $G \notin \mathcal{F}$ if and only if every block of $G$ is a cycle or a cycle with exactly one chord or a theta graph and at most one block of $G$ is not a cycle.

Theorem 1.11.([6]) Let $G$ be a connected graph with $\delta=2$ and edge connectivity one. Then $G \notin \mathcal{F}$ if and only if there exists a cut edge $e$ of $G$ such that at least one component of $G-e$ is a graph, all of whose blocks are cycles. 
Lemma 1.12.([6]) Let $G$ be a 2-edge connected graph such that exactly one block of $G$ is either a cycle with exactly one chord or a theta graph and all other blocks are cycles. Let $v \in V(G)$. Then there exists a minimum graphoidal cover $\psi$ of $G$ such that $v$ is the only vertex exterior to $\psi$ and there exists a path in $\psi$ which contains $v$ as an exterior vertex.

Theorem 1.13.([7]) Let $G$ be a 2-connected graph with $p \geq 3$. Then $G \in \mathcal{F}_{a}$ if and only if $G \notin \mathcal{G}(f)$.

Theorem 1.14.([7]) Let $G$ be a graph with $\delta>1$ and connectivity one. Then $G \notin \mathcal{F}_{a}$ if and only if at least one end block of $G$ is a member of $\mathcal{G}(f)$ with $f$ as a cut vertex of $\mathcal{G}$.

Theorem 1.15.([5]) Let $G \in \mathcal{G}(f)$ and $\Delta(G) \geq 3$. Let $v$ be a vertex of $G$ with $\operatorname{deg} v \neq \Delta$. Then there exists a minimum acyclic graphoidal cover $\psi$ of $G$ such that $v$ is the only vertex exterior to $\psi$ and $\eta_{a}=|\psi|=\Delta-1$.

\section{Main Results}

Lemma 2.1. Let $G$ be a graph whose blocks are all cycles. Let $u, v \in V(G)$ with $u \neq v$. Then there exists a graphoidal over $\psi$ of $G$ such that $u$ and $v$ are the only vertices exterior to $\psi$ and a path $P$ in $\psi$ which contains $u$ and $v$ as end vertices.

Proof. Let $C_{1}, C_{2}, \ldots, C_{k}$ be the blocks of $G$. By hypothesis each $C_{i}$ is a cycle, $i=1,2, \ldots, k$. Let $P$ be a $(u, v)$ - path in $G$. Let $S_{1}, S_{2}, \ldots, S_{s}, s \in\{1,2, \ldots, k\}$ be the segments of $P$ that are part of the cycles $C_{1}, C_{2}, \ldots, C_{s}$. Let $R_{i}=C_{i} \backslash S_{i}, 1 \leq i \leq s$. Then $\psi=\left\{P, R_{1}, R_{2}, \ldots, R_{s}, C_{s+1}, \ldots, C_{k}\right\}$ is a graphoidal cover of $G$ with $u$ and $v$ as the only vertices exterior to $\psi$.

Theorem 2.2. Let $G$ be a 2-edge connected graph. Then either $\eta=q-p$ or $\eta=q-p+1$.

Proof. Suppose $\eta \neq q-p$. By Theorem 1.10, every block of $G$ is a cycle or a cycle with exactly one chord or a theta graph and at most one block of $G$ is not a cycle. We now prove that $\eta=q-p+1$ by induction on $m$ where $m$ is the number of blocks of $G$. When $m=1, G$ is either a cycle or a cycle with exactly one chord or a theta graph. Clearly for any minimum graphoidal cover $\psi$ of $G$, exactly one vertex of $G$ is exterior to $\psi$ and hence by Theorem $1.3, \eta=q-p+1$.

We now assume that $\eta=q-p+1$ for all 2-edge connected graphs with $m$ blocks, $m \geq 1$. Let $G$ be a 2 -edge connected graph with $m+1$ blocks. Let $C$ be an end block which is a cycle and $v \in V(C)$ be a cut vertex of $G$. Let $G^{\prime}$ be the subgraph of $G$ obtained by removing all the vertices of $C-v$. Clearly $G^{\prime}$ has $m$ blocks. By induction 
hypothesis $\eta\left(G^{\prime}\right)=q^{\prime}-p^{\prime}+1$ when $p^{\prime}$ and $q^{\prime}$ are the order and size of $G^{\prime}$ respectively.

$$
\text { Now } \begin{aligned}
\eta(G) & =\eta\left(G^{\prime}\right)+1 \\
& =q^{\prime}-p^{\prime}+2 \\
& =q-p+1 . \text { This completes the induction and the proof. }
\end{aligned}
$$

Theorem 2.3. Let $G$ be a graph with $\kappa^{\prime}=1$ and $\delta>1$, where $\kappa^{\prime}$ is the edge connectivity of $G$. Let $S=\{e \mid e$ is a cut edge of $G$ and the bolcks of at least one component of $G$-e are all cycles $\}$. Let $\mathcal{H}_{G}=\left\{H_{1}, H_{2}, \ldots, H_{m}\right\}$, be the collection of all such components. Let $v_{i} \in V\left(H_{i}\right), 1 \leq i \leq m$. Then $\eta=q-p+m$ and there exists a minimum graphoidal cover $\psi$ of $G$ such that $v_{i}, 1 \leq i \leq m$ are the only vertices exterior to $\psi$ and there exist paths in $\psi$ which contain $v_{i}$ as an end vertex, $1 \leq i \leq m$.

Proof. Since contracting an edge incident with a cut vertex of degree 2 does not affect the value of $q-p$ and $\eta$, we may assume without loss of generality that any cut vertex has degree at least 3. We observe that if $m=0$, then the result follows from Theorem 1.11. Hence we assume that $m>0$ and prove the result by induction on $r$, where $r$ is the number of cut edges of $G$. Since $\kappa^{\prime}=1, r \geq 1$. Suppose $r=1$ and let $e=x_{1} x_{2}$ be the cut edge of $G$. Let $G_{1}$ and $G_{2}$ be the components of $G$-e with $x_{1} \in V\left(G_{1}\right)$ and $x_{2} \in V\left(G_{2}\right)$. Clearly $G_{1}$ and $G_{2}$ are 2-edge connected graphs. If both $G_{1}$ and $G_{2}$ are in $\mathcal{H}_{G}$ then by Lemma 2.1 there exists a graphoidal cover $\psi_{i}$ of $G_{i}$, $i=1,2$ and a $\left(v_{i}, x_{i}\right)$-path $P_{i}$ in $G_{i}$ such that $v_{i}$ and $x_{i}$ are the only vertices exterior to $\psi_{i}, i=1,2$. Then by Theorem $1.3 \psi=\left(\psi_{1} \backslash\left\{P_{1}\right\}\right) \cup\left(\psi_{2} \backslash\left\{P_{2}\right\}\right) \cup\left\{P_{1}\right.$ o e minimum graphoidal cover such that $v_{1}$ and $v_{2}$ are the only vertices exterior to $\psi$. Thus $\eta=q-p+2$. Suppose $G_{1} \notin \mathcal{H}_{G}$. For $G_{2} \in \mathcal{H}_{G}$ we define $\psi_{2}$ as before. If $G_{1} \in \mathcal{F}$, then there exists a minimum graphoidal cover $\psi_{1}$ of $G_{1}$ such that all vertices of $G_{1}$ are interior to $\psi_{1}$. Then by Theorem $1.3 \psi=\psi_{1} \cup\left(\psi_{2} \backslash\left\{P_{2}\right\}\right) \cup\left(\right.$ e o $\left.P_{2}^{-1}\right)$ is a minimum graphoidal cover of $G$ with $v_{2}$ as the only vertex exterior to $\psi$, where $e o P_{2}^{-1}$ is a path in $\psi$ which contains $v_{2}$ as an end vertex. If $G_{1} \notin \mathcal{F}$, then since $G_{1} \notin \mathcal{H}_{G}$, by Theorem 1.10 exactly one block of $G_{1}$ is a cycle with exactly one chord or a theta graph and all other blocks are cycles. Hence by Lemma 1.12 there exists a minimum graphoidal over $\psi_{1}$ of $G_{1}$ and a path $P_{1}$ in $\psi_{1}$ with $x_{1}$ as its terminus such that $x_{1}$ is the only vertex exterior to $\psi_{1}$. Then $\psi=\left(\psi_{1} \backslash\left\{P_{1}\right\}\right) \cup\left(\psi_{2} \backslash\left\{P_{2}\right\}\right) \cup\left\{P_{1}\right.$ o e o $\left.P_{2}^{-1}\right\}$ is a minimum graphoidal cover of $G$ such that $v_{2}$ is the only vertex exterior to $\psi$ and $P_{1}$ o e o $P_{2}^{-1}$ is a path in $\psi$ which contains $v_{2}$ as an end vertex. Thus $\eta=q-p+1$. Hence the result is true for $r=1$.

We now assume that the result is true for all graphs with at most $r-1$ cut edges. Let $G$ be a graph with $r$ cut edges satisfying the conditions of the theorem. Let $e=x_{1} y_{1}$ be a cut edge of $G$ such that one of the components of $G-e$ is $H_{1}$. Let $G_{1}$ be the other component of $G-e$ and let $x_{1} \in V\left(G_{1}\right)$ and $y_{1} \in V\left(H_{1}\right)$. By Lemma 2.1 there exists a graphoidal cover $\psi_{1}$ of $H_{1}$ whose only exterior vertices are $y_{1}$ and $v_{1}$ and there exists a $\left(y_{1}, v_{1}\right)$-path $Q_{1}$ in $\psi_{1}$. Clearly $\delta\left(G_{1}\right)>1$. 
Case(i). $x_{1} \in H$ for some $H \in \mathcal{H}_{G_{1}}$

By induction hypothesis there exists a minimum graphoidal cover $\psi_{2}$ of $G_{1}$ whose only exterior vertices are $x_{1}, v_{2}, \ldots, v_{m}$ and there exists a path $R_{1}$ in $\psi_{2}$ with $x_{1}$ as its terminus and path in $\psi_{2}$ with $v_{1}$ as terminus, $2 \leq i \leq m$. Then $\psi=\left(\psi_{1} \backslash\left\{Q_{1}\right\}\right) \cup\left(\psi_{2} \backslash\left\{R_{1}\right\}\right) \cup\left\{P_{1}\right\}$ where $P_{1}=R_{1}$ o e o $Q_{1}$ is the required minimum graphoidal cover of $G$.

Case(ii). $x_{1} \notin H$ for all $H \in \mathcal{H}_{G_{1}}$

By induction hypothesis there exists a minimum graphoidal cover $\psi_{2}$ of $G_{1}$ whose only exterior vertices are $v_{2}, v_{3}, \ldots, v_{m}$ and there exists a path $\psi_{2}$ with $v_{i}, 2 \leq i \leq m$ as terminus. Now $\psi=\left(\psi_{1} \backslash\left\{Q_{1}\right\}\right) \cup \psi_{2} \cup\left\{e o Q_{1}\right\}$ is the required minimum graphoidal cover of $G$. This completes the induction and the proof.

Theorem 2.4. Let $G$ be a connected graph which is not a tree with $n$ pendant vertices, $n \geq 1$ and $\left|\mathcal{H}_{G}\right|=m, m \geq 0$. Then $\eta=q-p+m+n$.

Proof. We prove the result by induction on $n$, the number of pendant vertices in $G$. Let $\mathcal{H}_{G}=\left\{H_{1}, H_{2}, \ldots, H_{m}\right\}$. Let $v_{i} \in V\left(H_{i}\right), 1 \leq i \leq m$ where $H_{i} \in \mathcal{H}_{G}$. Suppose $n=1$. Let $v \in V(G)$ be such that $\operatorname{deg} v=1$ and $P=\left(v, u_{1}, u_{2}, \ldots, u_{k}, w\right)$ be a path in $G$ such that $\operatorname{deg} u_{i}=2$, for all $i, 1 \leq i \leq k$ and $\operatorname{deg} w>2$. Such a vertex $w$ exists because $G$ is not a path. Let $G_{1}=G \backslash\left\{v, u_{1}, u_{2}, \ldots, u_{k}\right\}$.

Case(i). $w \in H$ for some $H \in \mathcal{H}_{G_{1}}$

Then by Theorem 2.3, there exists a minimum graphoidal cover $\psi_{1}$ of $G_{1}$ such that $v_{1}, v_{2}, \ldots, v_{m}, w$ are the only vertices exterior to $\psi_{1}$ and there exists paths in $\psi_{1}$ which contains $v_{i}, 1 \leq i \leq m$ and $w$ as end vertices. Let $P_{1}$ be the path in $\psi_{1}$ with $w$ as its terminus. Then $\psi=\left(\psi_{1} \backslash\left\{P_{1}\right\}\right) \cup\left\{P_{1} \circ P^{-1}\right\}$ is a minimum graphoidal cover of $G$ such that $v_{1}, v_{2}, \ldots, v_{m}, v$ are the only vertices exterior to $\psi$ and $\eta=q-p+m+1$.

Case(ii). $w \notin H$ for all $H \in \mathcal{H}_{G_{1}}$

By Theorem 2.3, there exists a minimum graphoidal cover $\psi_{1}$ of $G_{1}$ such that $v_{1}, v_{2}$, $\ldots, v_{m}$ are the only vertices exterior to $\psi_{1}$. Hence $\psi=\psi_{1} \cup\{P\}$ is a minimum graphoidal cover with $v_{1}, v_{2}, \ldots, v_{m}$ and $v$ as the only vertices exterior to $\psi$. Hence $\eta=q-p+m+1$. Therefore the result is true for $n=1$.

We assume the result is true for $k$ pendant vertices. Let $G$ be a graph with $k+$ 1 pendant vertices and let $z_{1}, z_{2}, \ldots, z_{k+1}$ be the pendant vertices of $G$. Let $P=$ $\left(z_{1}, u_{1}, \ldots, u_{k}, w\right)$ be a path in $G$ such that $\operatorname{deg} u_{i}=2$, for all $i, 1 \leq i \leq k$ and $\operatorname{deg} w>2$. Let $G_{1}=H \backslash\left\{z_{1}, u_{1}, u_{2}, \ldots, u_{k}\right\}$. If $w \in H$ for some $H \in \mathcal{H}_{G_{1}}$, then by induction hypothesis there exists a minimum graphoidal cover $\psi_{1}$ of $G_{1}$ such that $v_{1}, v_{2}, \ldots, v_{m}, w, z_{2}, z_{3}, \ldots, z_{k+1}$ are the only vertices exterior to $\psi_{1}$ and there exists a path $P_{1}$ in $\psi_{1}$ with $w$ as its terminus. Hence $\psi=\left(\psi_{1} \backslash\left\{P_{1}\right\}\right) \cup\left\{P_{1} \circ P^{-1}\right\}$ is a minimum graphoidal cover of $G$ with $z_{1}, z_{2}, \ldots, z_{k+1}, v_{1}, v_{2}, \ldots, v_{m}$ as the only vertices exterior to $\psi$. Thus $\eta=q-p+n+m$. If $w \notin H$ for all $H \in \mathcal{H}_{G}$, then by induction hypothesis there exists a minimum graphoidal cover $\psi_{1}$ of $G_{1}$ such that $v_{1}, v_{2}, \ldots, v_{m}, z_{2}, \ldots, z_{k+1}$ are the only vertices exterior to $\psi_{1}$. Hence $\psi=\psi_{1} \cup\{P\}$ is a minimum graphoidal cover 
of $G$ with $v_{1}, v_{2}, \ldots, v_{m}$ and $z_{1}, z_{2}, \ldots, z_{k+1}$ as the only vertices exterior to $\psi$. Thus $\eta=q-p+m+n$.

The next theorem determines the acyclic graphoidal covering number of a graph with $\delta=2$.

Theorem 2.5. Let $G$ be a graph with $\delta=2$. Let $B_{1}, B_{2}, \ldots, B_{m}, m \geq 0$ be end blocks of $G$ which are in $\mathcal{G}\left(f_{i}\right)$ with $f_{i}$ as a cut vertex. Let $v_{i} \in V\left(B_{i}\right), 1 \leq i \leq m$ and $v_{i}$ is not a cut vertex of $G$. Then there exists a minimum acyclic graphoidal cover $\psi$ of $G$ whose only exterior vertices are $v_{1}, v_{2}, \ldots, v_{m}$ and $\eta_{a}=q-p+m$.

Proof. We observe that if $m=0$, then the result follows from Theorem 1.13 and Theorem 1.14. Hence we assume that $m>0$ and prove the result by induction on $n$, where $n$ is the number of blocks of $G$. If $n=1$, the result follows from Theorem 1.15. We now assume that the result is true for all graphs with at most $n-1$ blocks. Let $G$ be a graph with $n$ blocks satisfying the conditions of the theorem. Let $\psi_{1}$ be a minimum acyclic graphoidal cover of $B_{1}$ whose only exterior vertex is $v_{1}$. Let $H=G \backslash\left(V\left(B_{1}\right) \backslash\left\{f_{1}\right\}\right)$.

Case(i). $\operatorname{deg}_{H} f_{1}=1$.

We choose a path $P=\left(f_{1}, u_{1}, \ldots, u_{k}, w\right)$, such that $\operatorname{deg} u_{i}=2$ for each $i$ and $\operatorname{deg} w>$ 2. Let $G_{1}=H \backslash\left\{f_{1}, u_{1}, u_{2}, \ldots, u_{k}\right\}$. Clearly $\delta\left(G_{1}\right)>1$. Let $B$ be a block of $G_{1}$ containing $w$. If $B$ is an end block of $G_{1}$ and $B \in \mathcal{G}(f)$ with $f$ a cut vertex of $G$, then by induction hypothesis there exists a minimum acyclic graphoidal cover $\psi_{2}$ of $G_{1}$ whose only exterior vertices are $w, v_{2}, \ldots, v_{m}$. Let $P_{1}$ be a path in $\psi_{2}$ having $w$ as its terminus. Then $\psi=\left(\psi_{2} \backslash\left\{P_{1}\right\}\right) \cup\left\{P_{1} \circ P^{-1}\right\} \cup \psi_{1}$ is a minimum acyclic graphoidal cover of $G$ with $v_{1}, v_{2}, \ldots, v_{m}$ as its only exterior vertices.

Otherwise by induction hypothesis there exists a minimum acyclic graphoidal cover $\psi_{2}$ of $G_{1}$ whose only exterior vertices are $v_{2}, v_{3}, \ldots, v_{m}$ and $\psi=\psi_{2} \cup\{P\} \cup \psi_{1}$ is a minimum acyclic graphoidal cover of $G$ whose only exterior vertices are $v_{1}, v_{2}, \ldots, v_{m}$.

Case(ii). $\operatorname{deg}_{H} f_{1}>1$.

Let $B$ be a block of $H$ containing $f_{1}$. If $B$ is an end block of $G$ and $B \in \mathcal{G}(f)$ with $f$ as a cut vertex then by induction hypothesis there exists a minimum acyclic graphoidal cover $\psi_{2}$ of $H$ with $v_{2}, v_{3}, \ldots, v_{m}$ and $f_{1}$ as its only exterior vertices. Then $\psi=\psi_{1} \cup \psi_{2}$ is the required minimum acyclic graphoidal cover of $G$.

Otherwise by induction hypothesis there exists a minimum acyclic graphoidal cover $\psi_{2}$ of $H$ with $v_{2}, v_{3}, \ldots, v_{m}$ as its only exterior vertices. Let $P$ be the path in $\psi_{2}$ having $f_{1}$ as an internal vertex. Let $x, y$ be the terminal vertices of $P$. Let $P_{1}$ and $P_{2}$ be the $\left(x, f_{1}\right)$ and $\left(f_{1}, y\right)$ - sections of $P$ respectively. Then $\psi=\psi_{1} \cup\left(\psi_{2} \backslash\{P\}\right) \cup\left\{P_{1}, P_{2}\right\}$ is the required minimum acyclic graphoidal cover of $G$. This completes the induction and the proof.

Theorem 2.6. Let $G$ be a graph with $n$ pendant vertices, $n \geq 1$ and let $B_{1}, B_{2}, \ldots$, $B_{m}, m \geq 0$ be end blocks of $G$ which are in $\mathcal{G}\left(f_{i}\right)$ with $f_{i}$ as a cut vertex. Then $\eta_{a}=$ $q-p+m+n$. 
Proof. Similar to the proof of Theorem 2.3.

We now proceed to the main theorem of characterizing the class of graphs with $\eta=\eta_{a}$.

Remark 2.7. Since $\eta \leq \eta_{a}, \eta_{a}=q-p$ implies that $\eta=q-p$. Theorem 1.13 and Theorem 1.14 characterizes the class of all graphs for which $\eta_{a}=q-p$. Hence for these graphs $\eta=q-p$, in turn $\eta=\eta_{a}$. Hence we need to consider the case when $\eta_{a} \neq q-p$.

Theorem 2.8. Let $G$ be a connected graph with $\eta_{a} \neq q-p$ and $\delta \leq 2$. Then $\eta=\eta_{a}$ if and only if one of the following holds.

(i) If $G$ has no cut edge, then $G$ is a graph such that an end block of $G$ is either a theta graph or a cycle with exactly one chord whose vertices of degree 3 are not cut vertices and all other blocks are cycles and the block-cut point tree of $G$ is a path (Refer Figure 1).

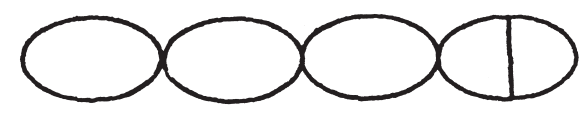

Figure 1.

(ii) If $G$ has a cut edge with $\left|\mathcal{H}_{G}\right|=m$ and if $l$ is the number of end blocks in $\mathcal{G}(f)$ with $f$ as a cut vertex, then $m=l$, where $\mathcal{H}_{G}$ is as defined in Theorem 2.3 (Refer Figure 2).

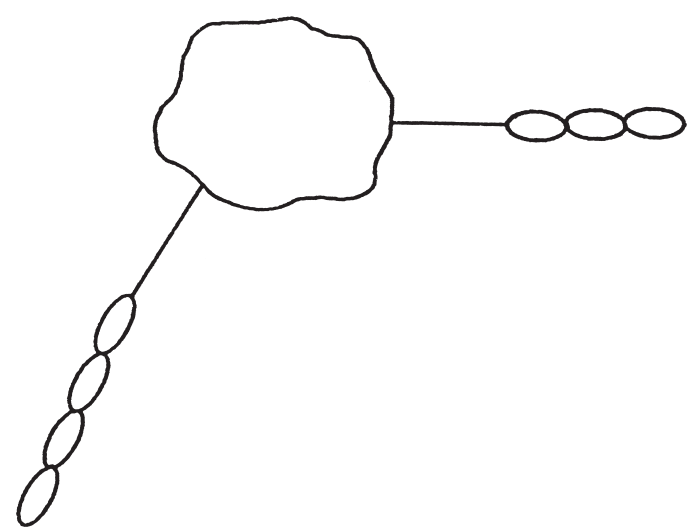

Figure 2.

Proof. If $G$ is of type (i) by Lemma 1.12 and Theorem 2.3, $\eta=\eta_{a}=q-p+1$. If $G$ is of type (ii), let $\left|\mathcal{H}_{G}\right|=m$ and let $n$ be the number of pendant vertices in $G$. Then by Theorem 2.4 and Theorem 2.6, $\eta=\eta_{a}=q-p+m+n$. Hence $\eta=\eta_{a}$. 
Conversely suppose $\eta=\eta_{a}$. We first prove the theorem when $\delta=2$. Since $\eta_{a} \neq q-p$, we have $\eta \neq q-p$. Hence by Theorem 1.9, Theorem 1.10 and Theorem 1.11, $G$ is either

(a) a block which is either a cycle or a cycle with exactly one chord or a theta graph, or

(b) a graph in which each block is a cycle or a cycle with exactly one chord or a theta graph and at most one block is not a cycle, or

(c) a graph which has a cut edge $e$ such that at least one component of $G-e$ is a graph whose blocks are all cycles.

If $G$ is of type (a) or (b) then by Theorem 2.2, $\eta=q-p+1$. Suppose $G$ is of type (a), a clock. If $G$ is a cycle then clearly $\eta \neq \eta_{a}$. Hence $G$ is a cycle with exactly one chord or a theta graph and $G$ reduces to a graph of type (i) given in the theorem. Suppose $G$ is of type (b), not a clock. Let $s$ be the number of end blocks of $G$ which are cycles. If each block of $G$ is a cycle then by Theorem 2.5, $\eta_{a}=q-p+s$. Since $\eta=\eta_{a}, s=1$ which is a contradiction to the fact that $G$ is not a block. Hence there exists a block $B$ in $G$ which is not a cycle. We claim that $s=1$. Suppose $s>1$. Then by Theorem 2.5,

$$
\eta_{a}= \begin{cases}q-p+s+1, & \text { if } B \text { is an end block of } G \text { and a vertex of } \\ & \text { degree } 3 \text { is a cut vertex of } G, \\ q-p+s, & \text { otherwise }\end{cases}
$$

Hence $\eta_{a}-\eta=s$ or $s-1$ and $s>1$ which is a contradiction to the fact that $\eta=\eta_{a}$. Hence $s=1$ and this proves that the block-cut point tree of $G$ is a path. If a vertex of degree 3 in $B$ is a cut vertex then again $\eta_{a}=q-p+2$ which is a contradiction. Hence vertices of degree 3 in $B$ are not cut vertices and $G$ reduces to a graph of type (i) given in the theorem.

If $G$ is of type (c), let $l$ be the number of end blocks in $G$ which are in $\mathcal{G}(f)$ with $f$ as a cut vertex and let $\left|\mathcal{H}_{G}\right|=m$. Then by Theorem 2.3 and Theorem 2.5, $\eta=q-p+m$, $\eta_{a}=q-p+l$. Since $\eta=\eta_{a}, m=l$. Thus $G$ reduces to a graph of type (ii) given in the theorem.

Now let $\delta=1$. Let $n$ be the number of pendant vertices of $G$. We define $l, m$ as before. By Theorem 2.4 and Theorem 2.6, $\eta=q-p+m+n$ and $\eta_{a}=q-p+l+n$. Since $\eta=\eta_{a}, l=m$. Thus $G$ reduces to a graph of type (ii) given in the theorem.

\section{References}

[1] B. D. Acharya and E. Sampathkumar, Graphoidal covers and graphoidal covering number of a graph, Indian J. Pure Appl. Math. 18(1987), 882-890.

[2] B. D. Acharya and Purnima Gupta, Domination in graphoidal covers of a graph, Discrete Mathematics 206(1999), 3-33. 
[3] S. Arumugam, B. D. Acharya and E. Sampathkumar, Graphoidal covers of a graph a cerative review., Graph Theory and Its Applications, Proceedings of the National Workshop, Manonmaniam Sundaranar University, Tirunelveli, Eds. S. Arumugam, B. D. Acharya and E. Sampathkumar, Tata-McGraw Hill (1997), 1-28.

[4] S. Arumugam, Indra Rajasingh, R. Bharathi and P. Roushini Leely Pushpam, On graph whose graphoidal covering number is one less than its cyclomatic number, Graph theory and Its Applications, Proceedings of the National Workshop, Manonmaniam Sundaranar University, Thirunelveli, Eds. S. Arumugam, B. D. Acharya and E. Sampathkumar, TataMcGraw Hill (1997), 29-34.

[5] S. Arumugam, Indra Rajasingh and P. Roushini Leely Pushpam, On graphs whose acyclic graphoidl covering number is one less than its maximum degree, Discrete Mathematics $\mathbf{2 4 0}(2001)$, 231-237.

[6] S. Arumugam, Indra Rajasingh and P. Roushini Leely Pushpam, A not on the graphoidal covering number of a graph, Journal of Discrete Mathematical Sciences and Cryptography 5(2002), 145-150.

[7] S. Arumugam, Indra Rajasingh and P. Roushini Leely Pushpam, On graphs whose acyclic graphoidal covering number is one less than its cyclomatic number - To appear in Ars Combinatoria.

[8] S. Arumugam, Indra Rajasingh and P. Roushini Leely Pushpam, Characterization of graphs with unique minimum graphoidal cover - To appear in Tamkang Journal of Mathematics, Tamkang University Republic of Chian.

[9] S. Arumugam, Indra Rajasingh and P. Roushini Leely Pushpam, Graphs with unique minimum acyclic graphoidal cover-I - To appear in the Journal of Discrete Mathematical Sciences and Cryptography.

[10] S. Arumugam and C. Pakkiam, The graphoidal covering number of hypergraphs, International Journal of Management and Systems 10(1994), 195-200.

[11] S. Arumugam and C. Pakkiam, Graphs with unique minimum graphoidal cover, Indian J. Pure Appl. Math. 25(1994), 1147-1153.

[12] S. Arumugam and C. Pakkiam, Graphoidal bipartite graphs, Graphs and Coimbinatorics 10(1994), 305-310.

[13] S. Arumugam and C. Pakkiam, The graphoidal covering number of directed graphs, J. Math. Phy. Sci. 29(1995), 33-41.

[14] S. Arumugam and J. Suresh Suseela, Acyclic graphoidal covering number of digraphs, Proceedings of the conferencee on Graph Connections, Cochin University, Cochin, Eds. R. Balakrishnan et al., Allied Publishers (1998), 72-78.

[15] S. Arumugam and J. Suresh Suseela, Acyclic graphoidal covers and path partitions in a graph, Discrete Mathematics 199(1998), 67-77.

[16] F. Harary, Graph Theory, Addison Wesley, Reading Mass, 1969.

[17] C. Pakkiam and S. Arumugam, On the graphoidal covering number of a graph, Indian J. Pure Appl. Math. 20(1989), 330-333.

[18] C. Pakkiam and S. Arumugam, The graphoidal covering number of unicyclic graphs, Indian J. Pure Appl. Math. 23(1992), 141-143.

[19] C. Pakkiam and S. Arumugam, The graphoidal graphs of a tree, Indian J. Pure Appl. Math. 21(1990), 1055-1058.

Department of Mathematics, Loyola College, Chennai - 600 034, India.

Department Mathematics, D. B. Jain College, Chennai - 600 096, India. 